\title{
Evolutionary Robot Swarm Cooperative Retrieval
}

\author{
Neil Vaughan ${ }^{1,2}$ \\ ${ }^{1}$ University of Chester, UK \\ ${ }^{2}$ Royal Academy of Engineering, London, UK \\ n. vaughan@chester.ac.uk
}

\begin{abstract}
In nature bees and leaf-cutter ants communicate to improve cooperation during food retrieval. This research aims to model communication in a swarm of autonomous robots. When food is identified robot communication is emitted within a limited range. Other robots within the range receive the communication and learn of the location and size of the food source. The simulation revealed that communication improved the rate of cooperative food retrieval tasks. However a counter-productive chain reaction can occur when robots repeat communications from other robots causing cooperation errors. This can lead to a large number of robots travelling towards the same food source at the same time. The food becomes depleted, before some robots have arrived. Several robots continue to communicate food presence, before arriving at the food source to find it gone. Nature-inspired communication can enhance swarm behaviour without requiring a central controller and may be useful in autonomous drones or vehicles.
\end{abstract}

Keywords: Swarm communication, Agents based modelling, Cooperative Retrieval, Robot simulation.

\section{Introduction}

Various animals including ants and bees communicate the location of food sources within groups. Leaf cutter ants generate multimodal communication signals in form of vibrations when cutting leaves which act as recruitment signals to other ants. Simulated models of insect navigation have been developed (Wystrach et al. 2016). Mammals such as mole rats use vocalization to communicate food presence.

For general purpose animal evolution, numerous evolutionary framework platforms were recently developed to enable study of virtual creature evolution to produce complex features combining previously evolved building blocks such as Avida (Lenski et al., 2003), (Devosoft, 2009) and Darwin Pond (Ventrella and Dodd, 2005). Noble Ape, 3DVCE, Tierra and Framsticks, Virtual models of living systems and artificial life evolution are growing in popularity within autonomous navigation research. Evolved virtual creatures (EVCs) within virtual reality environments provide an ideal platform for producing and testing methods of navigation and evolution (Sims, 1994) (Olson, 2013). Navigation in quadrupeds has recently been tackled (Coros et al., 2011).

Nature inspired algorithms led to recent multi-agent reinforcement learning. Image processing has been shown as useful for visual navigation (Vaughan, 2015). 
Other recent multi-agent reinforcement learning research simulating communication of swarm agents applied neuroevolution controllers for swarm agents (Vaughan, 2018).

\section{Methods}

For simplicity and speed, robots were modelled in 2D. Many available 3D robot robotic simulator tools currently model in 3D however the benefits of 3D are limited if the robots navigate on a flat ground. The robots were free to autonomously navigate. Each robot has numerous properties. Temporary properties include: $\mathrm{x}, \mathrm{y}$, angle, current state (see FSM) and quantity of food being carried. Other properties which can evolve over generations using a genetic algorithm are size, speed and colour. The robots had one memory location for location and size of food. The behaviour of each robot can be in one of a finite number of states within a finite state machine (FSM) (Fig. 1). The FSM diagram shows the states and transitions between states.

For swarm cooperation, when a robot identified food, the robot emitted a communication within a limited range. If any other robots are within the range they receive the communication. In this way the swarm can become aware of the location and size of the food source. The food location is stored in robot memory, overwriting memory of any smaller food (Fig. 2).

\section{Finite State Machine (FSM) Navigation}

Finite state machine was developed to define the changes between six different states: (1) walking, (2) standing, (3) turning, (4) looking for food, (5) going to a known food source and (6) returning to nest. Probabilities were defined as the chance of changing from one state to another (Fig. 1).

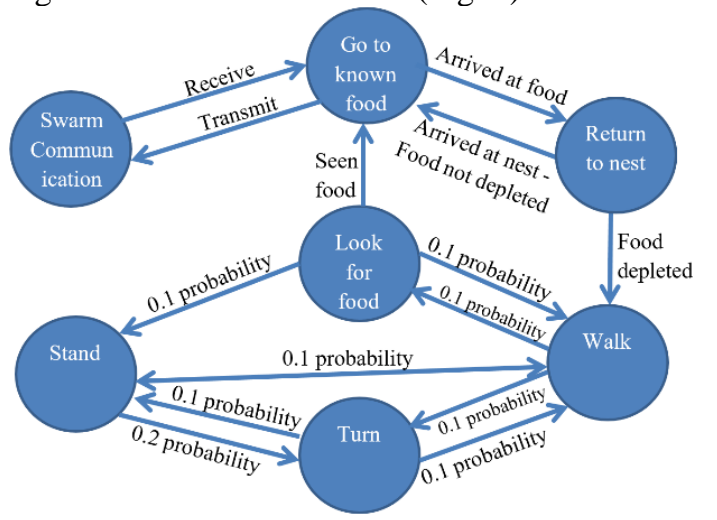

Fig. 1. Finite state machine controls autonomous navigation.

For collision detection, at each display time step, the screen is reloaded after each robot has been moved according to their current speed in their current direction. If a robot's new position would make it collide or overlap with the edge of the screen, or with another robot, they do not get moved and their position stays in the previous position. When 
robots and obstacles are circular, collision detection becomes a simple comparison of the distance between two robots compared to the sum of the robot radius. Collision detection was switched off during the communication simulation because it led to excessive congestion in larger swarms.

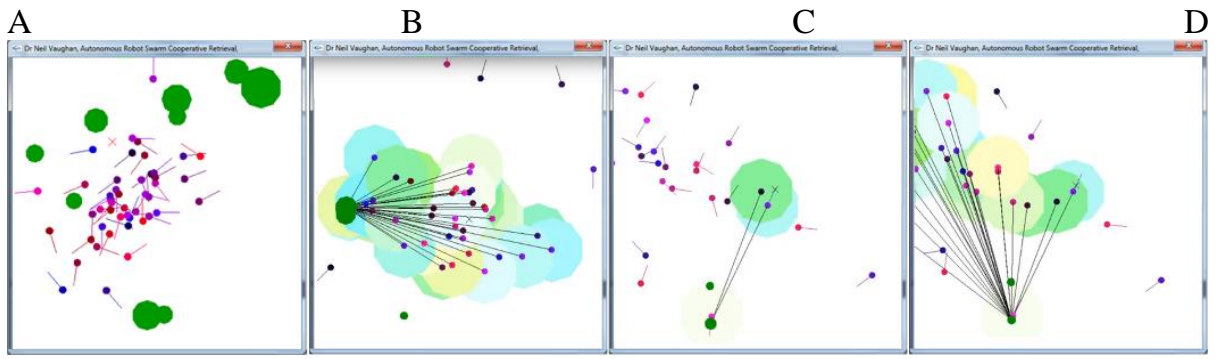

Fig. 2. Custom developed FSM cooperative swarm retrieval simulation. A) shows no communication, the agents locate food individually. B-D) shows with communication. B) All agents within distance $x$ converge on largest food, C) a purple agent found a small food, returned to the center to communicate, then D), agents within distance $x$ converge.

\section{$4 \quad$ Results}

The simulation revealed that individual non-communicating food retrieval (Fig. 2A) was out-performed by communicating agent retrieval (Fig. 2B-D). This was beneficial to increase the rate of cooperative tasks such as collecting and retrieving food to the nest when compared to. Fitness was measured as number of food collected over time.

Agent communications only propagate distance $x$ from the agent (Fig. 2C). Higher values of $x$ represent louder speech or better eyesight in audio or visual communication.

A video of the running simulation can be viewed at http://youtu.be/RKrceeOyykQ.

There can be re-communication problem as counter-productive chain reactions occur when robots repeat the communications they heard from other robots. This chain reaction effect reduced efficiency of cooperation. When a large number of robots travel towards the same food source at the same time, other potentially lucrative food sources are left and forgotten. In this case, the food source is used up quickly, yet some slower robots still had not arrived yet and believed that the food would still exist. They continued to communicate its presence erroneously, triggering other robots to follow, only to eventually arrive at the food source, to find it gone. This predicament could only be solved once all robots had arrived together at the food source to find it empty, which erased all memories of the food source.

Effect of memory limitations led to another finding that robots could benefit from having larger memory enabling them to store more than one food source at a time. In the limited memory situation where only one food source can be remembered, it commonly occurred that robots would forget a known food source in favour of a larger one communicated to them by another robot. Once the larger source was depleted, they had already forgotten about the original smaller source, which remained un-eaten for some further time, whilst the robot reverted to searching for food. If the robot had remembered the previous food in addition, it could have returned to get the first food after 
finishing the larger second food. A recursive approach could be proposed for food retrieval. However having extra memory could also cause longer delays between initially learning of a food source and eventually returning to it, which increases chances that the food may no longer exist due to other robots having heard the initial communications and already finished retrieving it.

\section{Conclusions}

Autonomous navigation research continues to rapidly increase with investments from many beneficiaries including autonomous vehicles, augmented smartphones, autonomous drones and autopilot technologies, autonomous robotics and computer game engines. Novel aspect of this research is a proposal for a new approach using simulated swarm communication within a virtual environment combined with finite state machine (FSM). This simulator approach provides a platform to develop and test algorithms for autonomous navigation which appropriately respond to sensory information in realtime. The study has revealed potential problems with unconstrained communication in swarms leading to inefficient cooperation in retrieval tasks. This could be remedied by adjustments to the structure of robots such as increasing memory.

\section{Acknowledgment}

The research was funded by The Royal Academy of Engineering (RAEng) as part of the Research Fellowship awarded to Dr Neil Vaughan as Principle Investigator.

The OpenGL source code in $\mathrm{c}$ and the compiled executable for windows are available to download: http://dec.bmth.ac.uk/ nvaughan/robot.

\section{References}

1. Coros et al (2011). "Locomotion skills for simulated quadrupeds." In ACM Transactions on Graphics (TOG), 30(4),ACM.

2. Devosoft (2009), Avida, http://avida.devosoft.org.

3. Ventrella J, Dodd B (2005). Darwin Pond, http://www.ventrella.com/darwin/darwin.html.

4. Lenski R.E., (2003). The Evolutionary Origin of Complex Features. Nature, 423:139-144.

5. Olson, R. et al. (2013). Evolved digital ecosystems: Dynamic steady state, not optimal fixed point. Adv. In ALife, ECAL. 12:126-133.

6. Sims, K. (1994). Evolving 3D morphology and behavior by competition. Artificial life, 1(4), 353-372.

7. Vaughan, N., 2015. Simulated Robotic Autonomous Agents with Motion Evolution. European Conference on Artificial Life ECAL.

8. Vaughan N, (2018) Swarm Communication by Evolutionary Algorithms, In IEEE Evolving and Adaptive Intelligent Systems (EAIS), 2018. IEEE.

9. Wystrach A, Dewar ADM, Philippides A, Graham P (2016) How do field of view and resolution affect the information content of panoramic scenes for visual navigation? A computational investigation. Journal of Comparative Physiology A, 202 (2). pp. 87-95. 